\title{
ANALYTICAL CHEMISTRY IN WATER POLLUTION CONTROL
}

\author{
K. H. MANCY \\ The University of Michigan, Ann Arbor, USA
}

\begin{abstract}
The most important, and yet frequently neglected, step in water pollution control programmes is the optimal design of the water quality measurement programmes. The design of water quality surveillance and monitoring programmes should follow a stepwise procedure, based on a logic scheme. This includes (a) definition of short and long-term objectives, (b) selection of parameters of analysis, and sites and frequency of measurement, and (c) selection of methods of analysis and data processing requirement. Perhaps the most important function in this protocol is the development of a preliminary model of the aquatic system under investigation capable of satisfying the measurement objectives. By means of recycling steps, the designer will be able to discard excess measurement and include missing information. Cost-effectiveness analysis may further modify the measurement programme and the derived model. Ultimately, the outcome of this design procedure will be optimized plans compatible with the measurement objectives and budget constraints phase.
\end{abstract}

\section{INTRODUCTION}

Water quality measurement for pollution control programmes is an extremely complicated and expensive task. It is imperative that great care be taken in the planning an optimizing of these programmes. The planning of measurement programmes cannot be left to the practised intuition of professionals in the field. An attempt is made in this paper to present a rational stepwise procedure for the optimal design of measurement systems. This subject is frequently ignored in the literature dealing with water quality analysis. The literature seems to be devoid of discussions concerned with the planning and the optimal design of measurement programmes. In contrast, the literature offers ample coverage of methods of analyses for various types of waters.

Depending on management objectives, water quality measurement programmes may take into account (a) the distribution dynamics of chemicals in the aqueous phase (soluble, colloidal or adsorbed on particulate matter); (b) accumulation and release by bottom deposits; (c) accumulation and release by aquatic biota; and (d) inputs from land and atmosphere, e.g. airborne particulates and land run-offs. A full characterization should take into account atmospheric interactions, e.g. evaporation, precipitation, currents and temperatures, as well as interactions with soil and sediments, e.g. siltation, leaching and erosion. 


\section{K. H. MANCY}

The successful implementation of water resources management is largely dependent on understanding this myriad of physicochemical and biological phenomena, and assembling this understanding into a coherent model. The utility of such models to planners of measurement programmes lies in its ability to identify missing information in terms of both type and quantity. Needless to say, the model will also identify information in excess and, hence, will assist in the optimization of the measurement programme.

This presentation offers only a brief overview. Relevant examples are given with special reference to the United Nations Development Programme and World Health Organization assisted water pollution programme in Poland. For further details the reader is advised to refer to the literature cited.

\section{DESIGN OF MEASUREMENT SYSTEMS}

A stepwise procedure for the optimal design of measurement systems is given in the flow diagram shown in Figure 1. The proposed design scheme

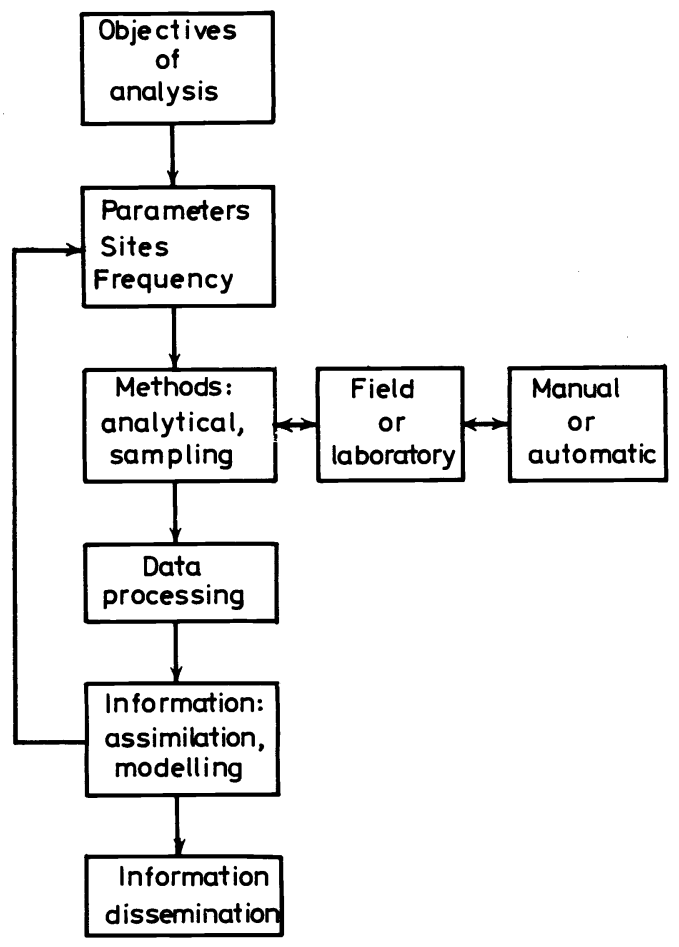

Figure 1. Flow diagram-design of measurement systems.

is based on eight primary steps : 


\section{ANALYTICAL CHEMISTRY IN WATER POLLUTION CONTROL}

1. Objectives: Why is measurement needed?

2. Parameters: What are the key parameters to be measured?

3. Sites: Where will measurement be made?

4. Frequency: What is the frequency of measurement?

5. Methods: How will measurements be made?

6. Data processing: What are the plans for data gathering, storage, retrieval and display?

7. Information assimilation: How will the data be assimilated to provide a model of the actual environment specific to the defined objectives in 1 ? (Recycle to 2, 3 and 4). At this point the designer will be able to determine if certain information is lacking or in excess. Consequently, it may be necessary to recycle to 2,3 and 4 in order to redefine the parameters of analysis, discard or add new ones. Similarly, it may be necessary to redefine the sites and quantity of measurement.

8. Information dissemination: This final step constitutes the appropriate design of methods for communicating the generated information in a way to meet the requirement of the users. The users should (a) see such information as potentially useful, and (b) understand how to apply this information within the context of their own self-defined interest, and also in a context in which their social, economic, and political agencies function.

\section{Objectives of measurement}

The first step in the design of measurement systems is the development of a clear statement of the objectives of measurement. This includes definitions of (a) short-term and long-term objectives of the resources management programme, (b) particular problems to which solutions are sought, and (c) limitations on the time required for programme implementation.

Some of the more common objectives of natural and waste waters analysis are as follows: (1) determine the suitability of water for an intended use; (2) determine treatment requirements for an intended use; (3) assess possible detrimental effects of waste effluent discharge on the quality of the receiving water; (4) assess and optimize treatment processes; (5) determine quantities of valuable byproducts which could be recovered from a waste effluent; and (6) conduct monitoring programmes to provide information on current and future water quality and quantity.

The last of the above-mentioned objectives may give the impression that this is nothing but 'measurement for the sake of measurement'. On the contrary, collection of information on current water quality and quantity, without having a specific problem in mind, constitutes the basis for enforcement programmes for the maintenance of water quality criteria and standards. Needless to say, this provides the necessary information for future assessment of impact resources exploitation.

In addition, these survey and monitoring programmes are essential for the control of hazardous material spills into water-courses. This may occur as a sudden discharge of toxic matter resulting from malfunction, collision, fire or human error in connection with rail, highway, and water modes of transportation or with stationary sources such as manufacturing or storage facilities. The hazardous material may be discharged directly into a water- 


\section{K. H. MANCY}

course, while in other cases the spilled material may flow or be washed into sewers or drainage channels or percolate into ground water supplies.

In addition to a clear definition of the purpose and goals of measurement, it is important to define (a) the geographical scope of the measurement system and (b) the system duration or a preliminary assessment of the duration of the measurement programme. Based on the objectives of measurement, it should be possible to define the geographical scope or the area included in the measurement system. For example, in the case of river surveillance it will be necessary to indicate whether to include the whole river basin or a basin sub-set. It may be necessary to limit measurement to certain sections of the river basin due to (a) state or national boundaries or (b) irrelevance to the purpose of the measurement. A map defining the geographical scope of the measurement programme should also indicate all effluent sources and tributary input points.

It is also necessary to approximate, as early in the design scheme as possible, the duration in terms of days, months, years, etc., of the required measurement. Depending on the objective of analysis, this period of time may be (a) anticipated by the designer or (b) simply dictated by administrative, legal or financial reasons. It will also be necessary to define the period of the year when measurement will be made if the system duration is not continuous throughout the year as it is less than one year.

\section{Parameters}

The next step in the design of measurement systems is the selection of parameters of analysis. This constitutes the types and levels of parameters required for the implementation of measurement objectives.

The choice of parameters for analysis should be done solely to satisfy the objectives of measurement. The designer should (a) gather information on water quality criteria and tolerance limits, or treatment performance characteristics, etc.; (b) develop an inventory of all thermal and material input to the water body under investigation, including tributaries and domestic and industrial outfalls; (c) identify parameters of particular interest for the purpose of measurement and prescribed water use; (d) specify threshold levels for each parameter at which violation is considered to have occurred; and (e) based on historical data, or preliminary measurement, specify the parameters selected for measurement in terms of their risk in violating the standards.

Selection of a given parameter is not sufficient without indicating the concentration levels for which measurement is required. This is significant in view of the fact that completely different procedures may be used for the sampling and analysis for a given constituent in the milligram per litre and microgram per litre ranges.

Parameters of analysis may be described as specific or non-specific. The latter often signifies a given property of a group of substances such as biochemical oxygen demand. Some of the more frequently used non-specific parameters are given in Table 1.

The selected parameters should also signify different degrees of specificity. For example, it may be necessary to specify the parameter as 'total phos- 
ANALYTICAL CHEMISTRY IN WATER POLLUTION CONTROL

Table 1. Non-specific water quality parameters

\begin{tabular}{lll}
\multicolumn{1}{c}{ Physical } & \multicolumn{1}{c}{ Chemical } & Physiological \\
\hline Filtrable residues & Hardness & Colour \\
Salinity & Alkalinity & Odour \\
Electrical conductance & BOD & Taste \\
Suspended matter & COD & \\
Turbidity & TOC & \\
& Chlorine demand & \\
\hline
\end{tabular}

BOD, biochemical oxygen demand.

COD, chemical oxygen demand.

TOC, total organic carbon.

phorus' or orthophosphate, polyphosphate and organophosphorus compounds.

Because water-soluble chemicals present the greatest threat to aquatic ecosystems from a counter-measure point of view, a priority ranking system for estimating the theoretical inherent hazards of these chemicals was prepared by the US Environmental Protection Agency ${ }^{1}$. The ranking system is based on (a) the lowest concentration range at which a material impairs any of the beneficial uses of water, (b) the quantity shipped annually by each mode of transport and (c) the probability of an accidental spill to surface waters for each transport mode. Since a material's ranking is then representative of its potential threat to water quality, it is then appropriate to use this system to provide guidelines in the selection of parameters of analysis, with specific reference to hazardous soluble substances. To illustrate this parameter ranking system, the top twenty hazardous substances are arranged in order of decreasing priority in Table 2.

Table 2. Parameter ranking priority for hazardous substances

\begin{tabular}{clcl}
\hline Rank & \multicolumn{1}{c}{ Substance } & Rank & \multicolumn{1}{c}{ Substance } \\
\hline 1 & Phenyl & 11 & Acetone cyanohydrin \\
2 & Methyl alcohol & 12 & Chlorine \\
3 & Cyclic rodenticides & 13 & Nonyl phenol \\
4 & Acrylonitriles & 14 & DDT \\
5 & Chlorosulphonic acid & 15 & Isoprene \\
6 & Benzene & 16 & Xylene \\
7 & Ammonia & 17 & Nitrophenol \\
8 & Misc. cyclic insecticides & 18 & Aldrin-taxophene group \\
9 & Phosphorous pentasulphide & 19 & Ammonium nitrate \\
10 & Styrene & 20 & Aluminium sulphate \\
\hline
\end{tabular}

For the purpose of analytical quality control of treatment processes, selection of parameters is usually based on process performance characteristics. The selection of parameters for establishing efficiency of treatment processes often takes into account operational guidelines for transient and steady state conditions.

\section{Site and frequency}

Selection of sites and determination of frequency of measurement will be 


\section{K. H. MANCY}

dealt with in greater detail below. For quantitative treatment of methods of selection of sites and determination of frequency of measurement in river survey programmes, the reader is referred to the report prepared by Beckers, Chamberlain and Grimsrud ${ }^{2}$.

The main factors which govern the selection of sites and frequencies of measurement may be summarized as follows:

\section{(1) Hydrodynamic properties}

The hydrodynamic properties are a major factor influencing site and frequency selection. In the case of rivers, streams, channels and to a lesser extent pipelines, it is important to specify the exact location of the measurement site within a given cross section. Depending on the flow characteristics and the extent of lateral mixing at any given location, it may be necessary to define each site in terms of depth from the surface and distance from either bank.

Frequency of measurement will be also governed by the prevailing hydrodynamic characteristics. The rates of physicochemical and biochemical transformations in aquatic environments are greatly influenced by the degree of mixing and water flowrate. Flocculation, precipitation, dissolution, adsorption, primary and secondary productivity, etc., are all dependent on hydrodynamics. Consequently, the frequency of measurement at any given site should take into account both (a) the flowrate and (b) the changes of flowrate with time.

Similarly, the selection of sites and frequency for measurement in lakes, estuaries and reservoirs should be guided by the prevailing water circulation patterns.

\section{(2) Environmental conditions}

Climatic conditions such as temperature, precipitation, wind speed and direction, etc., may have profound effects on water quality. The selection of sites and frequency of measurement should take into account these variables. This is usually done by predicting reasonable changes and modifying measurement sites and frequency accordingly.

\section{(3) Material input and decay}

Selection of measurement sites and frequency should take into account (a) geographical locations of point sources of discharge of heat and/or matter into the water body, (b) the rate of discharge in terms of type and quantities per unit time, and (c) changes in the concentration of the discharged material in the receiving water as a function of time and distance from the source of discharge. In river survey programmes this will include an account for all inputs from tributaries and municipal and industrial outfalls. Elaborate schemes for river segmentation which take into account material input and decay have been reported elsewhere ${ }^{2}$. Remote sensing using serial surveillance has been found to be quite useful in the identification of outfalls and the selection of measurement sites ${ }^{3-6}$.

After selecting the measurement parameters, sites and frequency of analysis, the designer should start identifying the methods of measurement. 


\section{Methods}

In the majority of water quality measurement programmes the selection of methods of analysis is usually left to the practised intuition of the analyst. In this discussion a number of basic guidelines are presented to assist the designer to the optimal choice of methods of sampling and analysis. In this section the author emphasizes the economic aspects of measurement and the need for cost-effectiveness analysis.

(1) Basic guidelines

Selection of measurement methods should be based on the following guidelines.

(a) What is the total number of analyses?

(b) What is the frequency and geographical scope of measurement?

(c) What is the required rapidity of analysis?

(d) What are the required sensitivity and detection limits?

(e) What is the required selectivity?

(f) What are the constraints on accuracy and precision?

An assessment of total number and frequency of analyses will serve to give a first estimate of the magnitude of the measurement programme. This information will help the designer to decide whether the analysis should be performed manually or by means of automated systems.

Similarly the required detection limit, selectivity, accuracy and precision, will help the analyst to decide whether the analysis should be done by manual, classical techniques or by advanced, instrumental methods. The required rapidity of analysis often dictates the method of analysis. If the results of analysis are needed within a few minutes, this will certainly limit the choice of the method of analysis to one or two techniques.

The analyst is sometimes obliged to use a 'standard' or a 'referee' method. Listings of 'standard', and 'recommended' methods of analysis can be found in a variety of publications sponsored by several private and governmental agencies $^{7-14}$. In addition, in several instances industry has found it desirable to formalize listings of more specific methods for analysis of particular types of waste waters ${ }^{14}$.

While the application of uniform procedures is highly useful, the analyst must be careful to guard against overreliance upon such procedures, and against the possibility of being lulled into a false sense of security in instances where these procedures may not be applicable. Indiscriminate application of general purpose methods of analysis without due consideration of specific interferences and other problems must be avoided. It may be necessary to modify the standard or referee method to suit a particular application.

The above-mentioned basic requirements will give an insight into whether the analysis should be done in the laboratory or in the field. Laboratory methods may be based on manual or instrumental techniques. If the basic requirements for method selection specify low detection limits or high selectivity, accuracy and precision, it may be necessary to select an instrumental technique. For large numbers of measurements, it may be advisable to rely on automated systems such as the Technicon Autoanalyzer, to achieve high precision and reduce the cost of measurement. 


\section{K. H. MANCY}

Field analysis is based on either manual procedure, automatic water quality monitoring or remote sensing systems. Manual methods are essentially simplified laboratory procedures done on board ship, on trailers or on river banks ${ }^{15,16,17}$. Automatic water quality monitors utilize on-stream and in situ physicochemical sensor systems. Remote sensing techniques are primarily used for aerial surveillance of large areas of water resources for temperature, oil spills, suspended matter or fluorescent material. Detailed descriptions of the above-mentioned instrumental and automated methods can be found elsewhere ${ }^{3-6}$.

The next step is the determination of the availability of equipment and manpower. This is particularly significant in the case of instrumental and automatic techniques which require sophisticated equipment and trained personnel. The lack of such equipment or qualified personnel may ultimately result in the selection of manual methods for analysis which may offer less desirable performance characteristics.

\section{(2) Sampling programme}

It is recommended that the design of sampling programmes be done after selection of the methods of analysis. Too frequently the method of analysis dictates certain requirements on the sampling programme. These may include specifications on the method of sample collection, storage and transportation, or sample size, sample container or pretreatment requirements.

The significance of a chemical analysis is no greater than that of the sampling programme. Ideally speaking, a representative sample does not exist. Attempts are made, however, to come as close as possible to sample an aquatic environment without disturbing its physicochemical and biochemical characteristics.

The most important requirements for a satisfactory sample are that it be both valid and representative ${ }^{5}$. For a sample to be valid, it has to be one which has been collected by a process of random selection. Random selection is one of the most basic, yet most frequently violated, principles in development of a sampling programme. Any method of sampling that sacrifices random selection will impair statistical evaluation of the analytical data. If non-random sampling procedures are contemplated-perhaps for significant reasons of convenience-it is highly desirable to first demonstrate that the results of the analysis check with those which would be obtained by random sampling. This check would be essential prior to any statistical evaluation of the data.

A satisfactory sample is not only randomly drawn but also representative. This means that the composition of the sample should be identical with that of the water from which it was collected; the collected sample should have the same physicochemical characteristics as the sampled water at the time and site of sampling.

Planning for a sampling programme should be guided by the overall objectives of analysis, the parameters of analysis and the method of measurement. Main guidelines for the design of sampling programmes are given in Table 3.

One of the most important aspects of the sampling process is the care and preservation of the sample prior to analysis. This point cannot be over- 
ANALYTICAL CHEMISTRY IN WATER POLLUTION CONTROL

Table 3. Design of sampling programme

Validity of sample

Random sampling

Validity of systematic sampling

Validity of sampling frequency

Statistical considerations
Representativity of sample

Sampling sites

Sampling frequency and times

Sample size

Sample collection (manual or automatic)

Sample container

Sample transportation and storage

Data collected with each sample

emphasized. A water analysis is of limited value if the sample has undergone physicochemical or biochemical changes during transportation or storage. These changes are time dependent, but they usually proceed slowly. In general, the shorter the time that elapses between collection of a sample and its analysis, the more reliable will be the analytical results. Certain constituents may, however, require immediate analysis at the sample site.

Certain determinations are more sensitive than others to the method of handling of water samples before analysis. Changes in temperature and pressure may result in the escape of certain gaseous constituents (e.g. $\mathrm{O}_{2}, \mathrm{CO}_{2}$, $\mathrm{H}_{2} \mathrm{~S}, \mathrm{Cl}_{2}, \mathrm{CH}_{4}$ ), or the dissolution of some atmospheric gases (e.g. $\mathrm{O}_{2}$ ). It is recommended, therefore, that determinations for gases be done in the field, or to 'fix' such materials as oxygen, chlorine or hydrogen sulphide, the sample should be treated upon collection with stable oxidizing or reducing agent. It is also recommended that the temperature and $\mathrm{pH}$ of the water be determined at the site of sampling. Changes in temperature and $\mathrm{pH}$ may cause changes in the solubility of dissolved gases and certain non-volatile constituents, resulting in their separation from aqueous phase. Carbonic acidbicarbonate-carbonate equilibria may be shifted to release gaseous carbon dioxide, or to precipitate certain metal carbonates. Similarly, shifts in hydrogen sulphide-sulphide equilibria due to changes in $\mathrm{pH}$ and/or temperature may result in the escape of hydrogen sulphide or the precipitation of metal sulphides.

Heavy-metal ions may undergo a variety of physicochemical transformations during sample handling. It has been recommended that for analysis for $\mathrm{Al}, \mathrm{Cr}, \mathrm{Cu}, \mathrm{Fe}, \mathrm{Mn}$ and $\mathrm{Zn}$, samples should be filtered at the site of collection and acidified to about $\mathrm{pH} 3.5$ with glacial acetic acid. Acidification tends to minimize precipitation, as well as sorption on the wall of the sample container. Since acetic acid may stimulate growth of moulds, it may be necessary to add a small quantity of formaldehyde to the sample as a preservative.

Another major point of interest for handling water samples is the effect of biological activity on the sample characteristics. Microbiological activity may be effective in changing the nitrate-nitrite-ammonia balance, in reducing sulphate to sulphide, in decreasing the dissolved oxygen content, BOD, organophosphorus compounds and any readily degraded organic compound. Freezing of water samples is helpful in minimizing changes due to biological activity. Certain chemical preservatives, such as chloroform or formaldehyde, are sometimes added to water samples for this purpose. 
(3) Cost analysis

After selection of the methods of analysis and sampling, the designer should provide a cost analysis. This should include (a) manpower, (b) equipment, (c) supplies and (d) construction, purchase or renting of facilities. A close assessment of the cost of sampling should be made since this may constitute the larger portion of the total cost of the measurement programme.

The designer should also be aware of the availability of private and government agencies which provide analytical services. Such agencies usually have listings of the cost of the analysis of a given parameter per sample. Most of these agencies do not perform any sampling and it is the responsibility of the designer to specify the desired method of analysis.

A cost analysis, as approximate as it may be, may finally serve to decide between two or more possible methods of analysis. In other cases, the estimated cost of analysis may be prohibitive; consequently less adequate and more economical measurement procedures have to be chosen.

\section{Terminology and expression of results}

\section{(1) Expression of results}

One of the main problems facing water quality analysts nowadays is a lack of uniformity in nomenclature and methods of expression of results. An example of the present confusion of expressing results can be found in the many ways dissolved oxygen measurements are reported, i.e. in units of milligrams per litre, part per million, percentage saturation, partial pressure and oxygen tension. It is realized that it may be difficult or undesirable to express parameters always in the same units. Nevertheless, agreement should be reached on uniformity of expression of results for any given parameter.

It is recommended that the terminology and units of expression of results proposed by the International Organization of Standardization (ISO) be adopted $^{18,19}$. One of the main advantages in achieving uniformity of nomenclature and expression of analytical data is the ability to compare results from different measurement programmes. In addition, the adoption of the ISO recommendations will make it possible for scientists and engineers in other fields to understand and use results from water quality measurement programmes. Presentation of analytical results should be accompanied by a statistical treatment of the data. The key statistical concepts for evaluation of results are accuracy and precision. We all understand accuracy to mean a measure of how close analytical results approximate the 'true' value. An accurate and/or unbiased analysis is one which will average out to the true value if run a sufficient number of times. Biased analysis is one which will average out to a result which differs from the true value.

Consequently, precision of results is a measure of how well repeated tests agree with one another, regardless of whether or not they may represent the 'true' value. Also, under the general title of precision are the terms 'reproducibility' and 'repeatability'. Reproducibility is often measured by how well the analyses check when run by different people, at different times. Repeatability represents how close together the results are in repeat tests at the same time, on the same setup, with the same operators.

Deviations from the 'true' values of results can be due either to 'random' 


\section{ANALYTICAL CHEMISTRY IN WATER POLLUTION CONTROL}

or 'systematic' errors. Random errors are always present in any measurement and are characterized by a systematic dispersion of deviations from the true value, described by the 'normal distribution'. The 'standard deviation' has been largely used as a measure of precision. This should give a reliable estimate since it is an essential parameter of the theoretical distribution of error.

Systematic errors do not necessarily exist. They are characterized by unidirectional deviations from the 'true' value. In this case the analysis is described as being biased in one direction. Corrections can be made to account for systematic errors. That is why they are frequently described as determinate errors. Small systematic errors are sometimes difficult to detect in the presence of large random errors.

Accuracy determinations should represent the combined systematic and random errors of results. Consequently, high accuracy should mean a small total of errors. An important distinction between precision and accuracy is that accurate measurements are always precise; the converse is not necessarily true. The precision of a series of measurements may be good, but every result may be higher than the true value because of an unsuspected interference. Only when systematic errors are minimized will precision also imply accuracy.

Statistical techniques can also be used for the rejection of outlying observations and plotting of results. The reader is advised to consult treatments by Youden ${ }^{20}$, Dixon and Massey ${ }^{21}$, Wilson ${ }^{22}$, Mandel $^{23}$ and Montgomery and Hart $^{24}$.

\section{(2) Graphical representations}

Graphical representation of analyses depends primarily on the objectives of analysis. A considerable number of techniques have been proposed. Graphical representations should be done for a purpose, i.e. to provide means for comparing results of analyses or to emphasize differences and similarities. Graphical procedures do this much more effectively than numbers quoted in tables.

Most graphical displays are designed to represent concentrations of various parameters, or a function of time, location, environmental factors, etc. These may be described simply as 'concentration diagram', where the data are represented by vertical bars, parallel lines, etc. Types of concentration diagrams include 'pie', circular, triangular, rectangular and three-dimensional displays. A survey of the different types of concentration diagrams and illustrations of their use has been reported by $\mathrm{Hem}^{25}$.

A useful procedure in the display of water quality characteristics is to enter the information on a map of the area under investigation. One form of water quality map is prepared by entering the information as symbols at each sampling point. The symbol can be a bar graph, a pattern diagram or a distinctive colour tracing along the stream. Isogram maps are frequently used for mapping groundwater quality. They are characterized by drawing lines of equal concentrations of given parameters. The validity of this technique is dependent on the homogeneity of water composition in the vertical direction at the sampling points.

Symbols indicating water quality criteria are frequently used, especially 


\section{K. H. MANCY}

with steam classification. Classification can be based on biological or chemical parameters, or a combination of both.

\section{Assimilation of information}

In this step of the development of the measurement programme, results of analyses are assimilated to describe the system under investigation. It is only at this time that it will be possible to determine certain information is lacking or certain information is in excess. If certain information is lacking, this will require recycling to select new parameters, methods of analyses, sampling, etc., as shown in Figure 1. Similarly, if certain information is in excess, this will require recycling to redefine the parameters of analysis and discard the ones judged to be unnecessary.

The assimilation of the analytical results is essentially a system modelling process. The process involves (a) choice of relationships among chosen parameters (system components), (b) identification of mathematical equations which describe these relationships and (c) application of these expressions using real data. The developed model should approximately describe the system under investigation in context with the measurement objective. It will be possible at this stage to detect gaps of information in the model which may warrant the redesigning of the measurement programme.

Data assimilation into a water quality model will serve to optimize the measurement programmes and offer an insight to the most direct way of gathering the needed information. It will also serve to screen the data and assemble them in a readily accessible form.

\section{Dissemination of information}

This is the final step in the design of measurement programmes. In this step a decision is made on how to present the gathered information. This may take different forms, depending on the potential users of the information. Information dissemination should satisfy the requirements of the user. Consequently, the users should (a) see such information as potentially useful and (b) understand how to apply this information. The disseminated information should satisfy the users' self-defined interest in a context in which their social, economic and political agencies function.

\section{REFERENCES}

1 US Environmental Protection Agency, Control of Spillage of Hazardous Polluting Substances, Report No. 15090 FOZ, prepared by Battelle Memorial Institute, Pacific Northwest Laboratories, EPA Contract No. 14-12-866, November (1970).

2 C. V. Beckers, S. G. Chamberlain and G. P. Grimsrud, Quantitative Methods for Preliminary Design of Water Quality Surveillance Systems, US Environmental Protection Agency Report R5-72-001 (1972).

3 K. H. Mancy, Instrumental Analysis for Water Pollution Control. Ann Arbor Science Publishers: Ann Arbor, Michigan (1971).

4 K. H. Mancy and W. J. Weber Jr, Analysis of Industrial Wastewaters. Wiley-Interscience: New York (1971).

5 K. H. Mancy, Analytical Problems in Water Pollution Control. National Bureau of Standards Special Publication 351. US Department of Commerce: Gaithesburg, Md. (1972). 


\section{ANALYTICAL CHEMISTRY IN WATER POLLUTION CONTROL}

6 H. E. Allen and K. H. Mancy, 'Design of Measurement Systems for Water Analysis', in Handbook of Water and Water Pollution (L. L. Ciacco, editor), Vol. 3, pp 971-1020 American Society of Testing Materials: Philadelphia (1972).

7 American Society of Testing Materials, Manual on Industrial Waste Waters, 2nd edition, American Society of Testing Materials: Philadelphia (1969).

8 American Public Health Association, American Water Works Association, and Water Pollution Control Federation, Standard Methods for the Examination of Water and Wastewater, 13th edition American Public Health Association (1971).

9 Institute of Water Engineers, the Royal Institute of Chemistry, the Society of Analytical Chemistry and the Society for Water Treatment, Approved Methods for Physical and Chemical Examination of Water, 3rd edition, London (1960).

10 Council for Mutual Economic Assistance, Standard Methods for Water Quality Examination, Moscow (1966).

11 German Methods for the Examination of Wàter, Wastewater and Sludge, Fachgruppe Wasserchemie in der Gesellschaft Deutscher Chemiker. Verlag Chemie: Weinheim (1960).

12 International Biological Program, Methods for Chemical Analysis of Fresh Waters, IPB Handbook No. 8 (1969).

13 Environmental Protection Agency (USA), Methods for Chemical Analysis of Water and Wastes (1969).

14 Manuals Prepared by Industry (USA), 8.1 American Petroleum Institute, 8.2 Soap and Detergent Association, 8.3 Pulp and Paper Industry.

15 F. H. Rainwater and L. L. Thatcher, Methods for Collection and Analysis of Water Samples, US Geological Survey Water Supply Paper 1454 (1960).

16 R. S. Ingols, 'A mobile river monitor', Water and Sewage Works, Nov. (1967).

${ }_{17}$ M. R. Soderquist and D. W. Taylor, 'A mobile laboratory for food processing', Water and Sewage Works, Ref. Number R-85-R89 (1972).

18 International Organization for Standardization, Recommendation R78, Geneva, Switzerland (1969).

19 International Organization for Standardization, Recommendation No. 1777, Geneva, Switzerland (1971).

20 W. J. Youden, Statistical Methods for Chemists. Wiley: New York (1951).

21 W. J. Dixon and F. J. Massey, Introduction to Statistical Analysis. McGraw-Hill: New York (1957).

22 A. L. Wilson, Talanta, 17, 21 (1970).

23 J. Mandel, The Statistical Analysis of Experimental Data. Wiley: New York (1964).

24 H. A. C. Montgomery and I. C. Hart, The Design of Sampling Programs for Rivers and Effluents, paper presented at meeting of Metropolitan and Southern Branch of the Institute of Water Pollution Control, England (1972).

$25 \mathrm{~J}$. Hem, 'Study and interpretation of the chemical characteristics of natural waters', US Geological Survey Paper No. 1473, 256 (1970).

26 Proceedings of the Speciality Conference on Automatic Water Quality Monitoring in Europe (P. A. Krenkel, editor), held in Kraków, Poland, 2 April 1971. Technical Report No. 28, Vanderbilt University Press.

i7 Proceedings of Workshop on Design of Environmental Information Systems (R. A. Deininger, editor), held in Katowice, Poland, January 1973. Ann Arbor Science Publishers: Ann Arbor, Michigan.

\section{APPENDIX: CURRENT PRACTICES IN POLAND}

Poland has been one of the leading European countries in developing strong water quality measurement programmes. This was done with the assistance of United Nations Development Programmes (UNDP) executed by the Regional Office for Europe of the World Health Organization (WHO). These UNDP/WHO programmes provided special funds for the purchase of some of the most advanced and sophisticated analytical instruments and water quality monitoring equipment. Furthermore, the UNDP/WHO programmes provided support for the training of Polish scientists and engineers 
on water quality measurement and advanced monitoring and surveillance techniques. This was done by means of short courses conducted in Poland ${ }^{3}$, fellowship programmes abroad, and symposia and workshops ${ }^{26,27}$.

Extensive water quality measurement programmes are being conducted by a number of well-equipped central laboratories in Poland, e.g. the Environmental Pollution Abatement Centres in Katowice, Warsaw and Wrocław. This includes trace analysis of micropollutants, e.g. pesticide residues, heavy metals $(\mathrm{Hg}, \mathrm{Pb}, \mathrm{Cd}, \mathrm{Zn})$, phenols and detergents.

A series of automatic water quality monitoring stations have been established on main rivers in Poland, i.e. Odra and Vistula. These automatic monitoring stations continuously measure $\mathrm{pH}$, electrical conductance, turbidity, chlorides, dissolved oxygen, oxidation-reduction potentials and water levels, utilizing appropriate sensor systems. These stations are either located on barges - so that they can be easily moved from one location to another-or housed in a stationary building on the river bank. Detailed descriptions of these water quality monitoring systems have been reported in the Proceedings of the Speciality Conference on Automatic Water Quality Monitoring in Europe (WHO symposium), held in Kraków in $1971^{26}$.

Water quality monitoring of rivers in Poland has been conducted to control waste discharges and to protect water intakes of all major cities against accidental spills. For example, the water quality monitoring station, on the Vistula, upstream from Warsaw water intake, provides analyses of phenols, cyanide, ammonia, detergents, mercury, total organic carbon, and dissolved oxygen. This monitoring station also conducts continuous fish bioassay tests for the detection of toxic matter. 